\title{
Aberrant NHERF1 expression as a biomarker in lung carcinoma
}

\author{
Deborah Belchis $^{1 *}$, Nathan Cuka ${ }^{2}$, Neeraj Sharma ${ }^{3}$, Rajni Sharma ${ }^{2}$ and Christopher D. Gocke ${ }^{2}$
}

*Correspondence: dbelchi1@jhmi.edu

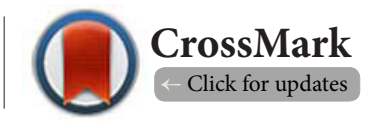

'Johns Hopkins Bayview Medical Center 4940 Eastern Avenue, Baltimore MD 21224, USA.

${ }^{2} J o h n s$ Hopkins University School of Medicine, 733 North Broadway, Baltimore, MD 21205, USA.

${ }^{3}$ McKusick-NathansInstitute of Genetic Medicine, Johns Hopkins University School of Medicine, 733 North Broadway, Baltimore, MD

21205, USA.

\begin{abstract}
Background: $\mathrm{Na} / \mathrm{H}$ exchange regulator factor 1 (NHERF1/EBP50) isan adaptor/signaling protein that when aberrantly expressed has been associated with tumor development and progression. In some tumors this occurs through its interaction with the PIK3-PTEN-AKT pathway.

Objective: This study explores NHERF1 biomarker expression in lung cancers and, further, NHERF 1 and PTEN expression and the PIK3CA-PTEN-AKT pathway in squamous cell lung carcinoma (SQCC).

Design: Phase 1: The immunohistochemical expression of NHERF1 was examined in a lung cancer microarray and correlated with histologic type of tumor and stage.Phase 2: NHERF1 and PTEN expression in $21 \mathrm{SQCC}$ was correlated. Mutations in the PIK3CA/PTEN/AKT pathway were analyzed by NGS.

Results and conclusions: Phase 1: NHERF1 expression and histologic subtype strongly correlated $(\mathrm{p}<0.001)$. Small cell carcinomas were negative. SQCCs exhibited moderate to strong expression. Strong aberrant expression in adenocarcinomas correlated with lymph node metastases $(p=0.005)$. Phase 2: 20 SQCC cases expressed NHERF1. PTEN was negative in 7 cases, heterogeneous in 2, and positive in 13. There was no correlation between NHERF 1 and PTEN expression, arguing against cooperativity of the proteins in this setting. AKT, PTEN, and PIK3CA did not have detectable mutations. PTEN loss was not associated with an underlying genetic mutation, suggesting a different mechanism for deregulation.
\end{abstract}

Keywords: Lung cancer, NHERF1, PIK3CA-PTEN-AKT pathway

\section{Introduction}

Lung cancer was the leading cause of cancer death in the US in 2015. It is the second most common new cancer diagnosed in the US, exceeded only by breast cancer [1]. While identification and targeting of actionable driver mutations has improved progression free survival in certain subsets of patients with adenocarcinoma of the lung, for the majority of lung cancer patients survival still remains poor [2]. In the subset of patients for whom targeted therapy is available and who do respond, the development of resistance has also become a major problem. For squamous cell carcinomas, targeted therapy has been elusive. These driver mutations, however, are only one component of complicated pathways which influence cell growth and proliferation. A better understanding of alterations in the entire set of pathways and other interacting components may provide additional therapeutic targets.

Many of these signaling pathways act through scaffolding proteins which bind various pathway components, creating complexes which then regulate signal transduction [3]. Na/H exchange regulator factor 1 (NHERF1/EBP50) is an adaptor/ scaffolding protein located at the apical membrane of cells and is an important regulator of transmembrane proteins and cytoskeletal structure and organization. It consists of 2 PDZ domains and an ezrin-radixin-moesin (ERM) binding region. Through these sites, NHERF1interacts with a variety of growth factors and regulatory proteins including EGFR and PDGFR (PDZ domains) and F-actin(ERM region) [4-9]. Recent studies of a variety of tumors have identified aberrant 
cytoplasmic, membranous, or nuclear expression of NHERF1 [5,10-13]. In studies of glioblastoma, breast and colon cancers, this delocalization from the normal apical membrane site and the appearance of aberrant expression has been correlated with

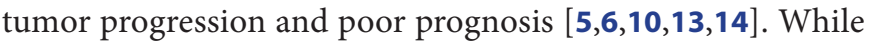
NHERF1 expression has been explored in variety of tumors, it hasnot been thoroughly examined in lung cancer. The initial phase of this study was to explore the expression of NHERF1 in different histologic subtypes of lung cancers and to correlate the findings with tumor stage.

The second stage of the study arose following the identification of relatively uniform aberrant cytoplasmic expression of NHERF1 in squamous cell carcinomas. One of the mechanisms by which NHERF1 is postulated to influence tumor development is through the PIK3CA/PTEN/AKT pathway $[\mathbf{5 , 1 5}$. Studies of NHERF1 in glioblastoma have demonstrated that NHERF1 interacts with the pleckstrin-homology domain leucine rich repeat protein phosphatases (PHLPP) and PTEN (PTEN-NHERF1-PHLPP) forming an inhibitory network on the PIK3CA-AKT pathway $[5,16,17]$. Disruption of the NHERF1-PDGFR or NHERF1PTEN complexes by either loss of NHERF1 or a shift from its normal apical location to the cytoplasm is postulated to lead to loss of the PTEN suppressor function and subsequent AKT activation [5]. As a subset of lung squamous cell carcinoma (SQCC) have been shown to harbor alterations in the PIK3CAAKT pathway, we decided in the second stage of the study to explore the possibility of an association between NHERF1 and PTEN immunoexpression. This was accompanied by mutation analysis of the PIK3CA-AKT-PTEN pathway, speculating that the increased expression of NHERF1 might be related to deregulation of that pathway.

\section{Materials and methods}

The study was approved by the Johns Hopkins Institutional Review Board.

\section{Histology}

Phase 1: A 99 cases/100 cores microarray consisting of a diverse assortment of lung cancers and benign conditions was purchased (product \#BC04002, Biomax, Rockville, MD). The cores were $1 \mathrm{~mm}$ in diameter and $5 \mathrm{um}$ thick. The array contained 20 cases of squamous cell carcinoma, 30 cases of adenocarcinoma, 10 cases of small cell carcinoma, 5 carcinoid tumors, 10 cases of metastatic carcinoma and 5 each of inflammatory pseudotumor, tuberculosis, and 5 of a mix of adjacent tissue near diseased tissue and normal tissue. For all cases the age and sex of the patient was provided. The histologic grade of the tumor, TMN classification, and total number of lymph nodes and the total number of positive lymph nodes were also given. None of the patients had metastatic disease (all were M0). Of the squamous cell carcinomas, the ages ranged from 41 to 69 . Seventeen were men and three were women. Seven cases were stage III, 5 were stage II, and 8 were stage I. Of the adenocarcinomas, the ages ranged from 41-71. Fifteen were men and 15 were women. Ten were stage III, 7 were stage II and 13 were stage I. Of the small cell carcinomas, the patients' ages ranged from 39 to 65 . Six were men and four were women. Three were stage III, 3 were stage II and 4 were stage 1 . Smoking history and molecular information was not provided on any of the cases.

Not all cores were readable. This study focused on the subtypes of pulmonary carcinoma. Of these, eleven squamous cell carcinomas, 29 adenocarcinomas, 10 small cell carcinomas, and 9 lymph node metastases were evaluable.

Phase 2: Twenty-one cases of squamous cell carcinoma from twenty patients were retrieved from the files of the pathology department at Johns Hopkins. Information was extracted from the clinical records including age, tumor stage, outcome, andimmunostaining results for any of the following confirmatory antibodies if performed at the time of diagnosis: p40, p63, TTF-1, or NAPSIN. Representative sections of each tumor were stained with NHERF1 and PTEN.

\section{Immunohistochemistry}

Immunostaining was developed using anti-rabbit NHERF1 polyclonal antibody (Pierce PA1-090, concentration $10 \mu \mathrm{g} / \mathrm{ml}$ ). Staining was performed on an automated Ventana immunostainer (BenchMark-Ultra, Ventana Medical Systems, Inc. Tucson, AZ) using UltraView DAB procedure. Briefly, slides were deparaffinized and hydrated and antigen retrieval was performed using a mild CC1 conditioner (high pH). Sections were incubated for 24 minutes with primary antibody and Ultra View detection (cat\# 760-500) was applied as per manufacturer's instructions. The slides were read independently by 2 pathologists and scored semiquantitatively from $0-3+$ as follows: staining intensity of the tumor cells was graded as 0 (negative), 1 (weak), 2 (moderate) and 3 (strong). The percentage of positive cells was graded as follows: $0,<10 \% ; 1,10-25 \% ; 2,26-50 \% ; 3,51-75 \% ; 4,76-90 \%$, and $5,>90 \%$. Apical staining was considered normal. Cytoplasmic, nuclear, or peripheral membranous staining were all considered abnormal patterns (Figure 1). A final score was derived from the combined intensity and percentage according to the method of Song et al. (Histopathology 2007, 51, 40-53). Samples with an intensity of 0 or 1 in $\leq 10 \%$ of cells were designated as negative, samples with an intensity of 1 in $>10 \%$ of cells or combined intensity and percentage scores of 2-3 were designated as weak, combined scores of 4- 6 were designated as moderate, and scores of 7-8 were designated as strong.

PTEN immunohistochemistry was performed on an automated Ventana platform (BenchMark-Ultra, Ventana Medical Systems, Inc. Tucson, AZ) using Opti-View detection system (Cat \# 760-700, (BenchMark-Ultra, Ventana Medical Systems, Inc. Tucson, AZ).ThePTEN antibody (Clone D4.3XP; Cell Signaling Technologies, Canvers, MA, dil 1:100) was applied for 36 minutes and detection was applied as per manufacturer's instructions. PTEN expression was assessed by comparison to the surrounding stromal components (fibroblasts and vascular structures). Negative was defined as absent protein expression with strong staining in surrounding stromal elements. Positive 
was considered diffuse nuclear and/or cytoplasmic staining present in similar intensity to surrounding normal stromal elements. Weak staining with less intensity than the surrounding tissue was categorized as heterogeneous [18] (Figure 2).

\section{Statistics}

Pearson's chi-square statistic was calculated for discrete variables (sex, stage) and one-way ANOVA for age, in comparison to score, with a Type 1 error of $5 \%$ for each. Fisher's exact test was performed for comparing NHERF1 expression in different histologic subtypes.

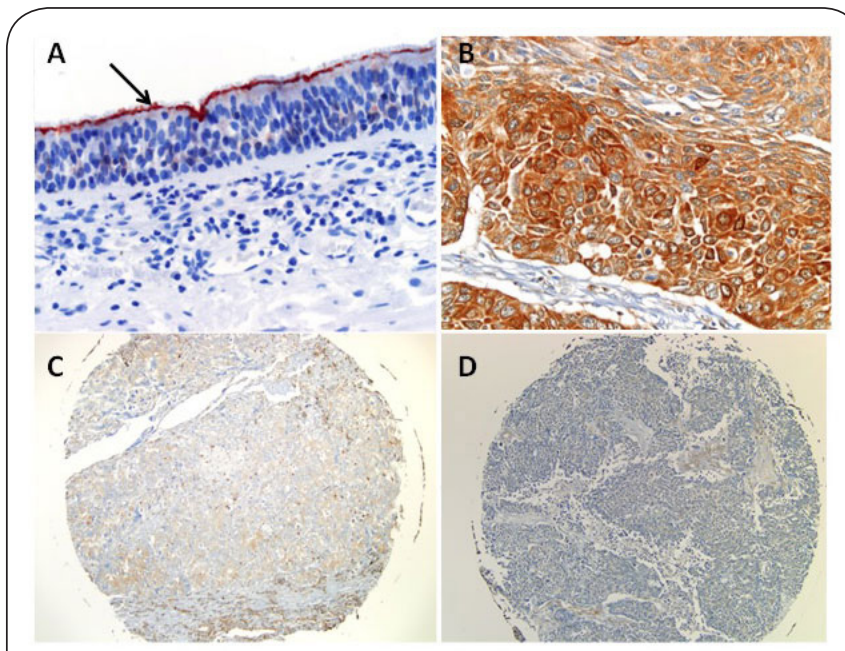

Figure 1. Patterns of NHERF1 staining in lung carcinoma. (A) Normal bronchial mucosa with apical staining of NHERF1 X200 (arrow), (B) Squamous cell carcinoma with strong cytoplasmic staining X400, (C) Adenocarcinoma with negative staining X100, (D) Small cell carcinoma with negative staining $\mathrm{X} 100$.

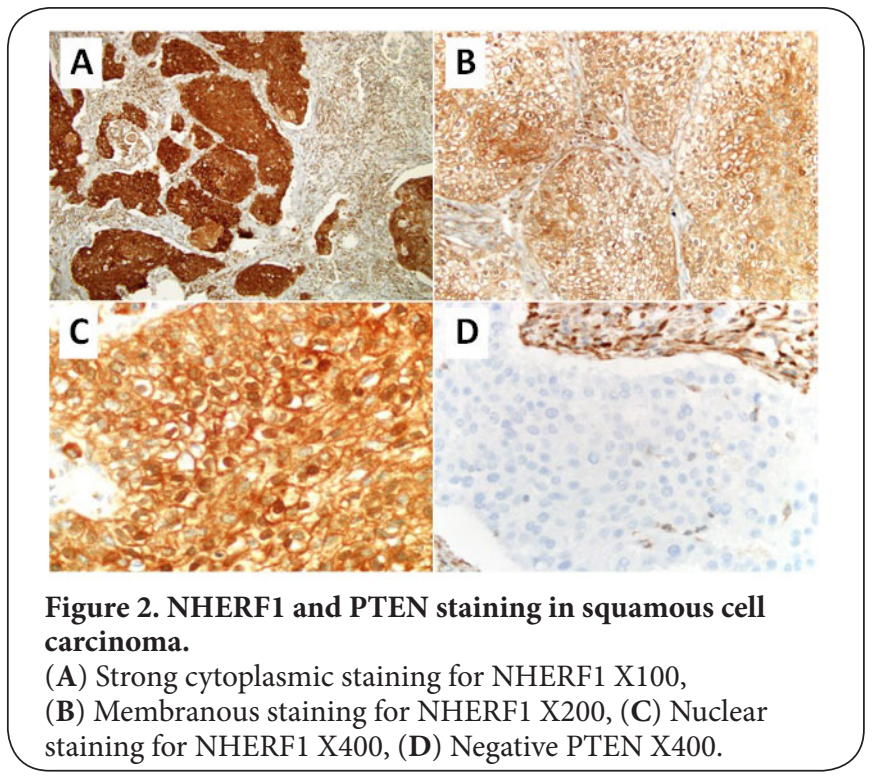

\section{Next generation sequencing (NGS)}

NGS was performed on each case as previously described [19]. Tumor in each case was manually micro-dissected and genomic DNA was extracted as described [20]. DNA sequencing of the frequently mutated regions of 50 cancer-related genes using the AmpliSeq Cancer Hotspot panel v.2 on the Ion Torrent PGM (Life Technologies, Inc, San Diego, CA) was performed as previously described [19]. This panel includes genes commonly mutated in the AKT/PIK3CA pathways.

\section{Statistics}

Statistical analysis was performed using Fisher's exact test with correction for small numbers.

\section{Results \\ Phase 1: Lung non-small cell carcinomas frequently express NHERF1}

Statistical analysis revealed a strong correlation between the histologic subtype and NHERF1 staining. The majority of the squamous cell carcinomas showed moderate to strong cytoplasmic staining in most cells compared to a range of staining in the adenocarcinomas and negative staining in the small cell carcinomas $(p<0.001)$. The adenocarcinomas' final scores for intensity and percentage of staining were as follows: $5 / 29$ cases (17\%) were negative, 5/29(17\%) were weak, $11 / 29(38 \%)$ were moderate, and $8 / 29(28 \%)$ were strong. The final scores for the squamous cell carcinomas were as follows: none were negative or weak, $2 / 11$ cases (18\%) were classified as moderate and $9 / 11(82 \%)$ as strong. Due to the small sample size, adenocarcinomas or squamous cell carcinomas with either a negative or weak final score were combined into one group and those with either a moderate or strong final score were combined into a second group. Each of these groups was then examined for correlation with stage and lymph nodes metastases, information which was provided by Biomax. Using this approach, moderate to strong expression of NHERF1 in adenocarcinomas correlated with the presence of lymph node metastases $(\mathrm{p}=0.0038)$ (Table 1). For the squamous cell carcinomas, there was no correlation of $T$ or N stage with NHERF1 expression (Table 1). Eight cases of squamous cell carcinoma metastatic to the lymph nodes were evaluable. Three were scored as negative and five received a final score of strong.

\section{Most non-small cell carcinomas exhibit aberrant cellular localization of NHERF1}

The patterns of expression were then evaluated. Twenty-four of the 29 adenocarcinomas had cytoplasmic staining (cNHERF1). Six of these showed concomitant nuclear staining (nNHERF1). None of the cases demonstrated membranous staining. Of the squamous cell carcinomas, 10 of 11 exhibited cytoplasmic staining, 2 had both cytoplasmic and nuclear staining, 2 exhibited membranous, cytoplasmic, and nuclear staining, and 1 showed membranous staining only.

Phase 2: The second part of the study focused on a relationship 
Belchis et al. Journal of Cancer Therapeutics \& Research 2016,

http://www.hoajonline.com/journals/pdf/2049-7962-5-7.pdf

doi: $10.7243 / 2049-7962-5-7$

Table 1. NHERF1 correlation with tumor stage, phase 1.

\begin{tabular}{lllll}
\hline & \multicolumn{2}{l}{ NHERF1 immunohistochemistry score } \\
\hline & Adenocarcinoma & \multicolumn{2}{l}{ Squamous cell } \\
\hline & Weak & Strong & Wegative/ & Moderate/ \\
& Seak & Strong \\
\hline Any T N0 & 9 & 6 & 0 & 6 \\
Any T N1\&2 & 1 & 13 & 0 & 5 \\
\hline
\end{tabular}

between NHERF1 and alterations in the PIK3CA-PTEN-AKT pathway. As alterations in the PIK3CA-PTEN-AKT pathwayare found in a significant subset of squamous cell carcinomas and NHERF1 is an integral member of the pathway, 21 cases of squamous cell carcinoma were analyzed for PTEN and NHERF1 expression. The clinical characteristics are listed in Table 2. In this phase, NHERF1 immunoexpression was correlated with the immunoexpression of PTEN and with mutations in the pathway including PIK3CA, PTEN, and AKT. NHERF1 staining wasscored as described above. Sixteen cases were given a strong score, 3 were moderate, 1 was weak and 1 was negative. The pattern was cytoplasmic in 20 of 21 cases. In addition, 5 showed membranous expression, 4 showed nuclear staining, and 2 cases had membranous, nuclear, and cytoplasmic expression. Immunohistochemical staining for PTEN revealed the following: PTEN was negative in 7 cases, heterogeneous in 2 , and uniformly positive in 13. Original and the recurrent tumors were available in one patient and both expressed PTEN and NHERF1. The 1 case without NHERF1 staining expressed PTEN. NGS showed no mutationsin AKT, PTEN, and PIK3CA. Most of the tumors were $\mathrm{T} 1$ or T2 and N0, therefore,statistical correlation of PTEN and NHERF with stage was not possible. No correlation was noted between PTEN and NHERF1 expression (Table 3 ) or between NHERF1 and mutations in the PIK3CA-PTEN-AKT pathway.

Table 2. Patient and tumor characteristics, phase 2.

\begin{tabular}{ll}
\hline & Number \\
\hline Age (mean [range]) & $70(55-81)$ \\
Sex (Male:Female) & $12: 10$ \\
Tumor Size (mean [range]) cm & $2.8(1.1-7.5)$ \\
Tumor Site (Right:Left) & $19: 5$ \\
\hline
\end{tabular}

\section{Discussion}

Our results show a strong correlation between aberrant NHERF1 expression and histologic subtype of tumor. Small cell carcinomas were uniformly negative for NHERF1 whereas squamous cell carcinomas showed almost uniform aberrant cytoplasmic expression. Adenocarcinomas showed a less consistent pattern, with $34 \%$ having no expression at all and the positive cases demonstrating a variety of aberrant localization patterns. In addition, we discovered an association between aberrant NHERF1 expression and the presence of lymph node metastases in adenocarcinomas. The only other published study of NHERF1 expression in lung cancer cases examined pleural effusion cy-
Table 3. Pathological findings, phase 2.

\begin{tabular}{lllllll}
\hline & & \multicolumn{3}{l}{ NHERF1 Score } & \multicolumn{3}{l}{ PTEN Score } \\
\hline TNM Stage & Number & S/M & W/N & P & H & N \\
\hline pT1-2N0 & 14 & 14 & 0 & 11 & 1 & 2 \\
pT3-4N0 & 3 & 2 & 1 & 1 & 0 & 2 \\
Any T N1-2 & 2 & 1 & 1 & 0 & 1 & 1 \\
Any T NX & 2 & 2 & 0 & 1 & 0 & 1 \\
\hline
\end{tabular}

S: Strong; M: Moderate; W: Weak; N: Negative; P: Positive; $\mathrm{H}$ : Heterogeneous

tology cell block samples of advanced lung cancers (stage IV) and surgical resection specimens (stage IA-IIIA) [21]. While that study showed delocalization of NHERF1 from the apical membrane to the cytoplasm in stage IA-IIIA surgical resection specimens, their study was significant for the loss of cNHERF1 and increased nNHERF1 in the effusion samples, suggesting a switch in the location of NHERF1 in more advanced stage cancers. We did not find this in our study. In phase one of our study, 6 of the adenocarcinomas and 4 of the squamous cell carcinomas exhibited both cytoplasmic and nuclear expression. Two of the adenocarcinoma cases and 2 of the squamous cell carcinoma cases were N0, proving that nuclear expression was not limited to high stage disease. Conversely, almost all low stage tumors maintain CNHERF1. This raises the possibility of a transitional state in which NHERF1 is expressed in both locations before being lost from the cytoplasm. Since we did not examine any stage IV tumors, we cannot confirm the existence of purely nNHERF tumors. Our results for stage I-III adenocarcinomas parallel those of non-lungcarcinomas where cytoplasmic expression of NHERF1 has been correlated with ag-

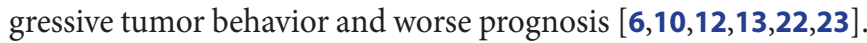

As indicated above, one mechanism by which NHERF1 is postulated to affect cellular proliferation and survival is through its interaction with members of the PIK3CA-PTEN-AKT pathway. However, we did not find a correlation between immunohistochemical (IHC) loss of PTEN and NHERF1 expression. PIK3CA alterations occur in non-small cell carcinomas. Studies have identified point mutations in $3.6 \%$ to $7.1 \%$ of squamous cell carcinomas and $1.5 \%$ to $2.6 \%$ of adenocarcinomas [24] although copy number variations are more common [24-27]. We did not find point mutations in PTEN or other members of the pathway but were unable to assay for copy number alteration. This suggests that in cases where PTEN was lost it occurred via a mechanism other than point mutation, such as epigenetic change. Other studies of PTEN expression in lung cancer have also noted that the frequent loss of PTEN expression by IHC analysis is not always associated with underlying genetic mutations, pointing to an alternative explanation for loss of protein expression [28-30]. Due to the small number of cases of squamous cell carcinoma with lymph node metastasis, the possibility that PTEN expression might be associated with stage cannot be excluded.

The significance of the cytoplasmic expression of NHERF1 
in squamous cell carcinomas is unclear. As a scaffolding protein it interacts with many different growth factors and signaling pathways ([7], including Yes-associated protein and TAZ. Further study of NHERF1 in these tumors may identify a pathway that can be exploited therapeutically.

\section{Conclusions}

NHERF1 is a key factor in signal transduction. Through its PDZ domains it interacts with growth factors and mitogenic signaling receptors. We found a statistically significant difference in NHERF1 expression between different histologic subtypes of lung cancer. Aberrant expression is essentially never seen in small cell carcinoma, whereas it is common in adenocarcinomas and almost always present in squamous cell carcinomas. In addition, adenocarcinomas show a range of staining with NHERF1 from weak to strong, with moderate to strong expression correlating with the presence of lymph node metastases in stage I-III tumors. Moderate to strong expression of NHERF1 is typically noted in squamous cell carcinoma of the lung. In addition, the loss of PTEN expression in squamous cell carcinoma does not correlate with an underlying genetic point mutation, implicating other mechanisms such as epigenetic changes.

\section{List of abbreviations}

NHERF1: $\mathrm{Na} / \mathrm{H}$ exchange regulator factor 1

NGS: Next Generation Sequencing

\section{Competing interests}

The authors declare that they have no competing interests.

\section{Authors' contributions}

D. Belchis conceived, designed, and executed the study, N. Cuka interpreted the pathology and analyzed the data, R. Sharma performed laboratory studies, N. Sharma wrote the paper, C. Gocke wrote paper and performed statistical analysis.

\section{Publication history}

Editor: Wei-Hsiung Yang, Mercer University School of Medicine, USA. Received: 02 May 2016 Revised: 16 June 2016

Accepted: 08 July 2016 Published: 18 July 2016

\section{References}

1. Siegel RL, Miller KD and Jemal A. Cancer statistics, 2016. CA Cancer J Clin. 2016; 66:7-30. | Article | PubMed

2. Camidge DR. Targeted therapy vs chemotherapy: which has had more impact on survival in lung cancer? Does targeted therapy make patients live longer? Hard to prove, but impossible to ignore. Clin Adv Hematol Oncol. 2014; 12:763-6. | PubMed

3. Luo LY and Hahn WC. Oncogenic Signaling Adaptor Proteins. J Genet Genomics. 2015; 42:521-529. | Article | PubMed Abstract I PubMed FullText

4. Pan $Y$, Wang $L$ and Dai JL. Suppression of breast cancer cell growth by $\mathrm{Na}+/ \mathrm{H}+$ exchanger regulatory factor $\mathbf{1}$ (NHERF1). Breast Cancer Res. 2006; 8:R63. | Article | PubMed Abstract | PubMed FullText

5. Molina JR, Agarwal NK, Morales FC, Hayashi Y, Aldape KD, Cote G and Georgescu MM. PTEN, NHERF1 and PHLPP form a tumor suppressor network that is disabled in glioblastoma. Oncogene. 2012; 31:1264-74. | Article I PubMed Abstract | PubMed FullText

6. Cardone RA, Greco MR, Capulli M, Weinman EJ, Busco G, Bellizzi A, Casavola V, Antelmi E, Ambruosi B, Dell'Aquila ME, Paradiso A, Teti A,
Rucci N and Reshkin SJ. NHERF1 acts as a molecular switch to program metastatic behavior and organotropism via its PDZ domains. Mol Biol Cell. 2012; 23:2028-40. | Article | PubMed Abstract | PubMed FullText

7. Voltz JW, Weinman EJ and Shenolikar S. Expanding the role of NHERF, a PDZ-domain containing protein adapter, to growth regulation. Oncogene. 2001; 20:6309-14. | Article | PubMed

8. Zheng JF, Sun LC, Liu H, Huang Y, Li Y and He J. EBP50 exerts tumor suppressor activity by promoting cell apoptosis and retarding extracellular signal-regulated kinase activity. Amino Acids. 2010; 38:1261-8. | Article | PubMed

9. Sizemore S, Cicek M, Sizemore N, Ng KP and Casey G. Podocalyxin increases the aggressive phenotype of breast and prostate cancer cells in vitro through its interaction with ezrin. Cancer Res. 2007; 67:6183-91. | Article | PubMed

10. Mangia A, Saponaro C, Malfettone A, Bisceglie D, Bellizzi A, Asselti M, Popescu O, Reshkin SJ, Paradiso A and Simone G. Involvement of nuclear NHERF1 in colorectal cancer progression. Oncol Rep. 2012; 28:889-94. | Article | PubMed

11. Malfettone A, Saponaro C, Paradiso A, Simone G and Mangia A. Peritumoral vascular invasion and NHERF1 expression define an immunophenotype of grade $\mathbf{2}$ invasive breast cancer associated with poor prognosis. BMC Cancer. 2012; 12:106. | Article | PubMed Abstract I PubMed FullText

12. Malfettone A, Silvestris N, Paradiso A, Mattioli E, Simone G and Mangia A. Overexpression of nuclear NHERF1 in advanced colorectal cancer: association with hypoxic microenvironment and tumor invasive phenotype. Exp Mol Pathol. 2012; 92:296-303. I Article I PubMed

13. Song J, Bai J, Yang W, Gabrielson EW, Chan DW and Zhang Z. Expression and clinicopathological significance of oestrogen-responsive ezrin-radixin-moesin-binding phosphoprotein $\mathbf{5 0}$ in breast cancer. Histopathology. 2007; 51:40-53. | Article I PubMed

14. Georgescu MM. NHERF1: molecular brake on the PI3K pathway in breast cancer. Breast Cancer Res. 2008; 10:106. I Article I PubMed Abstract | PubMed FullText

15. Takahashi Y, Morales FC, Kreimann EL and Georgescu MM. PTEN tumor suppressor associates with NHERF proteins to attenuate PDGF receptor signaling. EMBO J. 2006; 25:910-20. | Article | PubMed Abstract | PubMed FullText

16. Molina JR, Morales FC, Hayashi Y, Aldape KD and Georgescu MM. Loss of PTEN binding adapter protein NHERF1 from plasma membrane in glioblastoma contributes to PTEN inactivation. Cancer Res. 2010; 70:6697-703. | Article | PubMed Abstract | PubMed FullText

17. Cheng S, Li Y, Yang Y, Feng D, Yang L, Ma Q, Zheng S, Meng R, Wang S, Jiang WG and He J. Breast cancer-derived K172N, D301V mutations abolish $\mathrm{Na}+/ \mathrm{H}+$ exchanger regulatory factor 1 inhibition of plateletderived growth factor receptor signaling. FEBS Lett. 2013; 587:3289-95. | Article | PubMed

18. Bakkar RM, Xie SS, Urbauer DL, Djordjevic B, Vu K and Broaddus RR. Intact PTEN Expression by Immunohistochemistry is Associated With Decreased Survival in Advanced Stage Ovarian/Primary Peritoneal High-grade Serous Carcinoma. Int J Gynecol Pathol. 2015; 34:497-506. | Article | PubMed

19. Lin MT, Mosier SL, Thiess M, Beierl KF, Debeljak M, Tseng LH, Chen G, Yegnasubramanian S, Ho H, Cope L, Wheelan SJ, Gocke CD and Eshleman JR. Clinical validation of KRAS, BRAF, and EGFR mutation detection using next-generation sequencing. Am J Clin Pathol. 2014; 141:856-66. | Article | PubMed Abstract | PubMed FullText

20. Tsiatis AC, Norris-Kirby A, Rich RG, Hafez MJ, Gocke CD, Eshleman JR and Murphy KM. Comparison of Sanger sequencing, pyrosequencing, and melting curve analysis for the detection of KRAS mutations: diagnostic and clinical implications. J Mol Diagn. 2010; 12:425-32. | Article | PubMed Abstract | PubMed FullText

21. Mangia A, Partipilo G, Schirosi L, Saponaro C, Galetta D, Catino A, Scattone A and Simone G. Fine Needle Aspiration Cytology: A Tool to Study NHERF1 Expression as a Potential Marker of Aggressiveness in Lung Cancer. Mol Biotechnol. 2015; 57:549-57. I Article I PubMed

22. Bellizzi A, Greco MR, Rubino R, Paradiso A, Forciniti S, Zeeberg K, 
Cardone RA and Reshkin SJ. The scaffolding protein NHERF1 sensitizes EGFR-dependent tumor growth, motility and invadopodia function to gefitinib treatment in breast cancer cells. Int J Oncol. 2015; 46:1214-24. | Article | PubMed

23. Bellizzi A, Mangia A, Malfettone A, Cardone RA, Simone G, Reshkin $\mathrm{SJ}$ and Paradiso $\mathrm{A}$. $\mathrm{Na}+/ \mathrm{H}+$ exchanger regulatory factor 1 expression levels in blood and tissue predict breast tumour clinical behaviour. Histopathology. 2011; 58:1086-95. | Article | PubMed

24. Drilon A, Rekhtman N, Ladanyi M and Paik P. Squamous-cell carcinomas of the lung: emerging biology, controversies, and the promise of targeted therapy. Lancet Oncol. 2012; 13:e418-26. | Article | PubMed

25. Ji M, Guan H, Gao C, Shi B and Hou P. Highly frequent promoter methylation and PIK3CA amplification in non-small cell lung cancer (NSCLC). BMC Cancer. 2011; 11:147. | Article | PubMed Abstract | PubMed FullText

26. Okudela K, Suzuki M, Kageyama S, Bunai T, Nagura K, Igarashi $H$, Takamochi K, Suzuki K, Yamada T, Niwa H, Ohashi R, Ogawa H, Mori H, Kitamura H, Kaneko T, Tsuneyoshi T and Sugimura H. PIK3CA mutation and amplification in human lung cancer. Pathol Int. 2007; 57:664-71. | Article | PubMed

27. Yamamoto $H$, Shigematsu $H$, Nomura M, Lockwood WW, Sato M, Okumura N, Soh J, Suzuki M, Wistuba, II, Fong KM, Lee H, Toyooka S, Date H, Lam WL, Minna JD and Gazdar AF. PIK3CA mutations and copy number gains in human lung cancers. Cancer Res. 2008; 68:6913-21. | Article | PubMed Abstract | PubMed FullText

28. Sholl LM. Protein correlates of molecular alterations in lung adenocarcinoma: Immunohistochemistry as a surrogate for molecular analysis. Semin Diagn Pathol. 2015; 32:325-33. | Article | PubMed

29. Yanagawa N, Leduc C, Kohler D, Saieg MA, John T, Sykes J, Yoshimoto M, Pintilie M, Squire J, Shepherd FA and Tsao MS. Loss of phosphatase and tensin homolog protein expression is an independent poor prognostic marker in lung adenocarcinoma. J Thorac Oncol. 2012; 7:1513-21. | Article | PubMed

30. Soria JC, Lee HY, Lee JI, Wang L, Issa JP, Kemp BL, Liu DD, Kurie JM, Mao $L$ and Khuri FR. Lack of PTEN expression in non-small cell lung cancer could be related to promoter methylation. Clin Cancer Res. 2002; 8:1178-84. | Article | PubMed

\section{Citation:}

Belchis D, Cuka N, Sharma N, Sharma D and Gocke C.D. Aberrant NHERF1 expression as a biomarker in lung carcinoma. J Cancer Ther Res. 2016; 5:7. http://dx.doi.org/10.7243/2049-7962-5-7 